\title{
The design of power supply system based on WSN
}

\author{
Zhou xin ${ }^{1, a}$ \\ 1. Department of Communication Engineering, Chongqing College of electronic engineering, \\ Chongqing, 401331 \\ a.zhouxinkm@126.com
}

Keyword: WSN, senergy for node, solar-energy.

Abstract: Energy consumption of the node is one of the most limit of the development of the Wireless Sensor Network(WSN). and it is inevitable, so it's important to design a efficient and recyclable of energy system that to promote the development of the WSN. to the nodes in the WSN, the use of solar energy self -powered technology can effectively improve the service life of sensor nodes and reduce energy consumption a system that based on solar-energy is presented in this paper. The experiment shows that this system is of good reliability, and it can saving more energy, it also has a great value of practical application.

\section{Introduction}

The grow up of a new generation of computer network based on the internet of things which driving the rapid development of wireless sensor networks (WSN). Now the mature technology of wireless sensor networks mainly includes: infrared, Bluetooth, ZigBee and Wi-Fi. These wireless communication technologies have one thing in common, that is all of them need to enough power supply to the connected nodes. Because to most of the wireless sensor network, the number of nodes is very large, and the layout of the environment is relatively poor, such as the original forest, desert, and so on, thus causing the node's energy bottleneck. On the one hand, in order to control the cost of a single node, it is not able to carry large capacity power supply, on the other hand, they have to face the energy shortage caused by the consumption of the node.

In order to solve this problem, people begin to design and develop the self-powered technology. the main methods that can provide the power supply of Wireless sensor includes: solar cell, thermoelectric batteries, environmental electromagnetic wave power supply, etc. the existing solar power supply schemes mainly include the following:

Solar direct power supply scheme. this scheme consists of solar panel and a rechargeable lithium battery, this scheme has the advantages of its simple structure, but it will go continuous discharge of lithium battery charging, after repeated charge and discharge process of lithium battery, the storage capacity will be decrease [1].

Super capacitor scheme. in order to reduce the charging and discharging frequency of the lithium battery, a super capacitor is added between the solar panel and the lithium battery. The super capacitor can store a certain amount of power, when the voltage of the lithium battery is reduced to a certain value, the super capacitor will charge the lithium battery. although the maximum reduction of the charging frequency of the lithium battery, but in the final analysis ,it is still use lithium batteries to power the sensor nodes, and it can not solve the problem from the source at all [2].

In order to solve this problem, a scheme is designed in this paper, which combined with solar panels and lithium battery power supply, and reduce the application of lithium battery in the power supply system, in this way,it will prolong the service life of the lithium battery, by increasing the use cycle of the node, the wireless sensor network will be more stable.

\section{2 the overall scheme of power supply system}

The idea of this scheme is to use solar panels to supply power to the nodes as much as possible in the 
process of power supply. When there is sunlight, if the voltage pass through the solar panel reaches a certain threshold, it will supply power to the nodes with solar panels, under certain conditions, it can also give out a portion of the energy for charging the rechargeable battery. If the voltage pass through the solar panel is not able to supply power to the node, then the battery can supply power to the node. in this way, node can be the maximum possible continuous work. The scheme proposed in this paper mainly includes the following parts:

The power supply part. this part includes a solar battery board and a rechargeable battery. In the system, the solar panel is charged with the dual task of charging the rechargeable battery and supplying power for the sensor nodes. Rechargeable battery should be make the best possible choice of small size, light weight, charge and discharge more times .

Circuit part. this part includes a switch circuit, a charging management circuit, discharge protection circuit and a voltage stabilizing circuit. Switching circuit is mainly responsible for the direct power supply of solar panels and the choice of two modes of rechargeable batteries. Charge management circuit is responsible for the detection of battery voltage to prevent excessive battery charging. Discharge protection circuit can prevent excessive discharge of the battery caused by irreversible damage to the battery by detecting the voltage. Whether solar panels or rechargeable batteries, both of them can not fully meet the power supply requirements of the node, so it still need a voltage regulator circuit to regulate the node voltage to maximize the protection, the system block diagram of the design is shown in figure 1:

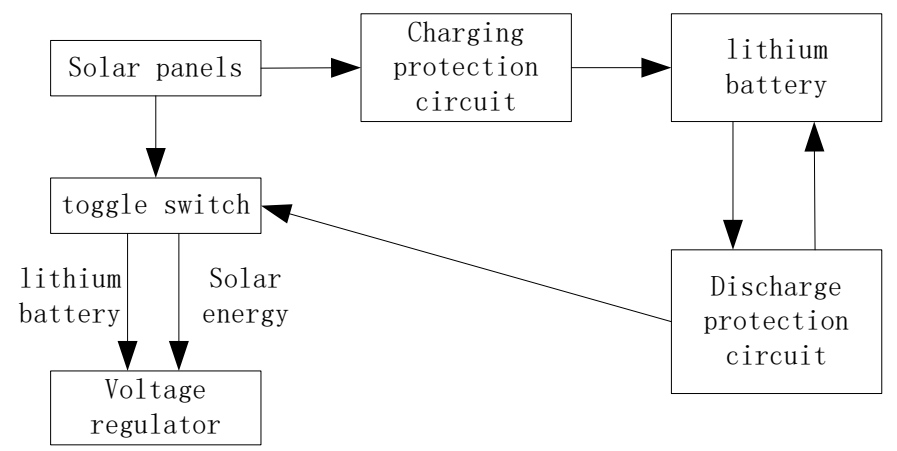

Figure 1. the system block diagram of the design

\section{3 selection of the power supply scheme}

Solar panels not only need to be responsible for the node power supply, but also for the battery charge, so it must be of enough power to achieve the load. This scheme adopts $135 \mathrm{~mm} * 125 \mathrm{~mm} * 4 \mathrm{~mm}$ polysilicon solar panels. The open circuit voltage of the solar panel can reach $7.5 \mathrm{~V}$, the nominal voltage is $6 \mathrm{~V}$, the maximum short circuit current is $300 \mathrm{~mA}$. Measured result shows that the maximum power of a single plate can reach $1.8 \mathrm{~W}$. Two solar panels are designed in parallel to form the solar cells of $12 \mathrm{~V}$ voltage, the maximum current is $600 \mathrm{~mA}$, and the power is $3.5 \mathrm{~W}$. Although the automatic installation platform can guarantee the maximum solar illumination angle to obtain the maximum conversion rate, but it has to give up this scheme for the high price and energy consumption to obtain the biggest benefit.

The choice of rechargeable batteries is $900 \mathrm{mAh}-083035$ polymer lithium battery. On the one hand, the quality of the lithium battery is light, the charging effect is good and can be charged and discharged for many times. After 500 times of charging, the battery can still keep 80\% of the energy. On the other hand, the peak current is $24 \mathrm{~mA}$ when the sensor node is received data, the peak current of the sending data is $29 \mathrm{~mA}$, and the working current of the sensor node is $30 \mathrm{~mA}$. For ease of calculation, the working current is calculated as $60 \mathrm{~mA}$ when the sensor node send or receive data, $900 \mathrm{mAh} / 60 \mathrm{~mA}=15 \mathrm{~h}$,. Therefore, the 
battery can continuously ensure that nodes work for 15 hours, due to the time of the node send or receive data is very short, the node is in a state of sleep in most of the cases, as a result, the battery can supply more than 45 days in full power.

\section{4 circuit design}

\section{1 switching circuit}

Switching circuit is the key part of the design, which can realize the power supply of lithium battery and the power supply of solar cell. The switching circuit adopts 8-bit serial successive approximation AD conversion chip ADC0832 and 8-bit CMOS microcontroller AT89S52 with high performance and low power consumption. The design of the circuit as shown in figure 2:

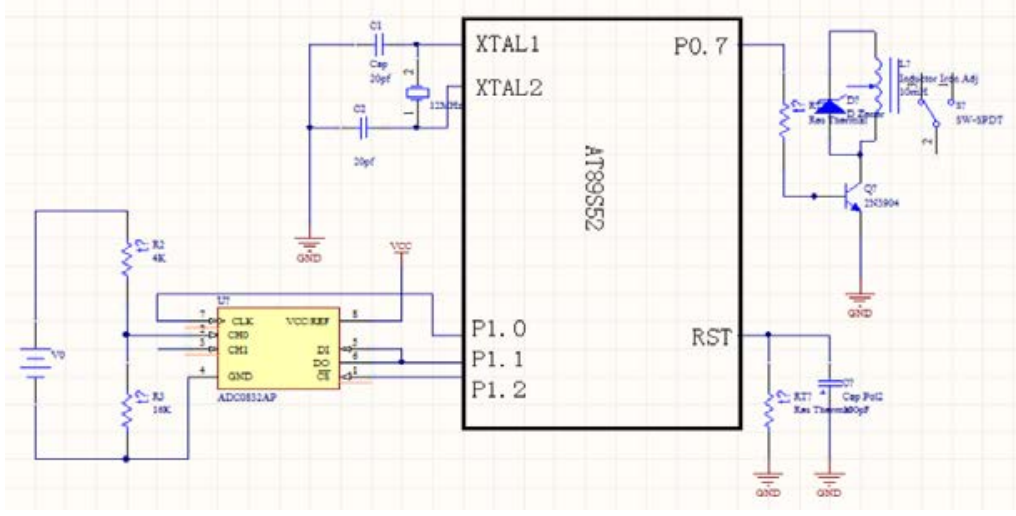

Figure 2.Switching circuit

As shown in Figure 2, assuming that in the case of a sufficient solar, the cell output voltage is V0, then the resistance voltage of R3 is V1. V1 is converted analog digital form by ADC0832, and sent to AT89S52 for analysis. In order to ensure the normal power supply of the node, the loss of power in the transmission is must to be taken into account, when the output voltage of the solar panel is not less than $3.7 \mathrm{~V}$,the system begin to use it. From the process of voltage distribution, it can be known:

$$
\mathrm{V} 1=\mathrm{V} 0 /(\mathrm{R} 2+\mathrm{R} 3) * \mathrm{R} 3=3.7 \mathrm{~V} /(4+16 \mathrm{~K}) * 16 \mathrm{~K}()=3 \mathrm{~V}
$$

That is only when detected the voltage $\mathrm{V} 1$ of the R3 is not less than $3 \mathrm{~V}$, the output voltage of the solar panel can reach 3.7V. V1 goes through the ADC0832, and was changed for the digital form, through the P1.2 port of AT89S52 for analysis. Since the D0 and D1 port can not be connected to the P1.2 port at the same time, so the D0 and D1 is connected together access to P1.2. then AT89S52 analysis of the digital form through the p0.7 port to control a relay. When the voltage is greater than $3 \mathrm{~V}$, the relay shifts its direction towards to the solar panels, using the solar panel to power supply; when the voltage less than $3 \mathrm{~V}$,the relay shifts the direction to the lithium batteries, lithium battery begins to supply power. Thus, the choice between solar panel power supply and lithium battery power supply is realized.

\section{2 charge management circuit}

Charging management circuit is common circuit of the power supply system, the main function of it is collecting energy and storaging into lithium batteries. At the same time charge management circuit is also responsible for the detection of the voltage of the lithium battery, when the voltage is below the predetermined value ,the system begins charging, When the charging is completed, the power charging is cut to protect the lithium battery from being over charged.

\section{3 discharge protection circuit}

In order to prevent the irreversible damage caused by the excessive discharge of the lithium battery, the discharge protection circuit is added in the system. Traditionally, a discharge protection circuit using a 
ADC channel to detect the voltage of the battery continuously, but in the process of analog to digital, a lot of energy will be consumed, in order to save energy effectively. The scheme uses Ricoh r5421 lithium battery protection chip for lithium batteries discharge detection protection. Under certain conditions, the chip can cut off the impulse and discharge of the battery, therefore the lithium battery can be protected.

\section{4 voltage stabilizing circuit}

Due to the discharge voltage of the using solar panels is $6 \mathrm{~V}$, and lithium battery discharge voltage is $3.6 \mathrm{~V} \sim 4.2 \mathrm{~V}$, while the node board ask for the higher voltage requirements, that is $3.3 \mathrm{~V}$ stably, so it is need to go through a voltage stabilizing circuit before entering the node board. In this design, the circuit is regulated by ASM1117, and the circuit diagram is shown in Figure 3:

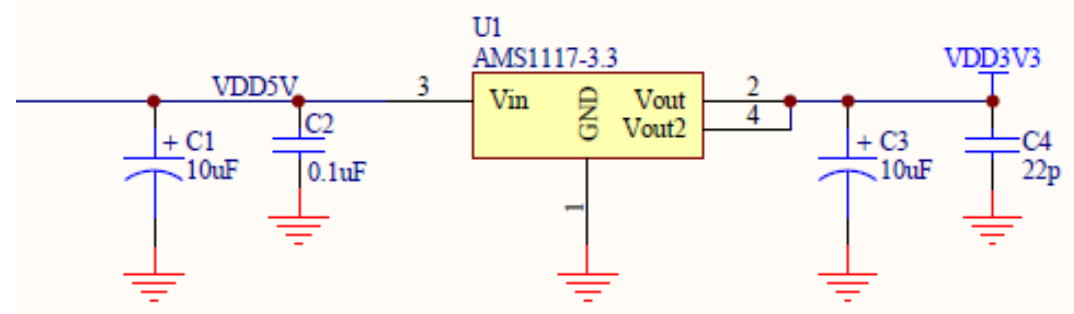

Figure 3. Voltage stabilizing circuit

\section{Conclusion}

In this paper ,a design of power supply system for WSN is presented, this design can realize the self powered based on solar energy. based on the traditional design of solar power supply system, dual power supply mode of direct power supply and rechargeable lithium battery powered by solar panel are proposed. when the voltage of the solar battery board reaches a certain threshold (in this design, the threshold is $3.7 \mathrm{~V}$ ) the node will be supplied by the solar panels directly, when the value is below the threshold, it was powered by a lithium battery. This design can reduce the using time of lithium battery and prolonging the life of the node.

\section{Acknowledgement}

This paper is supported by the fund of Sichuan Provincial Department of education research (SCYG2015-2-12)

\section{References}

[1] HU Qi-xun, DUAN Wei-jun, WANG Fu-bao, Design of Power System for Wireless Sensor Network Node Based on Solar Energy[J]. Modern electronics technique,2011,(2):23-26.

[2]ZHANG Qiang,YANG Tao. Self-powered Wireless Sensor Network for Environment Monitoring[J] .Instrument technique and sensor. 2008, (2):34-37.

[3]. YU Hong-yun ,LI Yan-qiu, SHANG Yong-hong, SU Bo. Design of a micro photovoltaic system for wireless sensor network[J]. Journal of functional materials and devices. 2008, 14(2):476-480

[4]. CUI Rong-qiang, Solar Photovoltaic Power: Hope of Low-Carbon Economy in China[J]. Chinese journal of nature. 2010, 32(3):149-154. 